\title{
Family-Based Tuberculosis Counseling Supports Directly Observed Therapy in Armenia: A Pilot Project
}

\author{
Nune Truzyan, Byron Crape, Tsovinar Harutyunyan, Varduhi Petrosyan \\ Zvart Avedisian Onanian Center for Health Services Research \& Development, Gerald and Patricia Turpanjian School of Public \\ Health, American University of Armenia, Yerevan, Armenia \\ Email: tnune@aua.am
}

How to cite this paper: Truzyan, N., Crape, B., Harutyunyan, T. and Petrosyan, V. (2018) Family-Based Tuberculosis Counseling Supports Directly Observed Therapy in Armenia: A Pilot Project. Journal of Tuberculosis Research, 6, 113-124. https://doi.org/10.4236/jtr.2018.62011

Received: March 15, 2018

Accepted: May 13, 2018

Published: May 16, 2018

Copyright $\odot 2018$ by authors and Scientific Research Publishing Inc. This work is licensed under the Creative Commons Attribution International License (CC BY 4.0).

http://creativecommons.org/licenses/by/4.0/

\section{(c) (i) Open Access}

\begin{abstract}
Objectives: Non-adherence to tuberculosis (TB) treatment causes development of multi-drug resistance (MDR). In Armenia, about $47 \%$ of previously-treated TB patients develop MDR-TB. This pilot intervention intended to explore the feasibility and effectiveness of a family-based-counseling (FBC) that included a psychological component in terms of improved adherence of TB patients to treatment and reduced stigma of TB. Methods: Overall, 136 regular TB patients (55) and their family members (81) participated in a single 90-minute interactive counseling session conducted in each household by the team of professional psychologist and TB nurse. To evaluate FBC effectiveness, we administered baseline and follow-up surveys to $52 \mathrm{~TB}$ patients and their 57 family members in 2012 and compared treatment outcomes of the study participants with the national data for 2011 and 2013. Results: We found that the intervention substantially improved the mean knowledge score of TB patients (from 19.2 to $21.6, \mathrm{p}<0.001$ ) and family members (from 18.0 to 21.7, $\mathrm{p}<0.001$ ). Percent of study participants thinking that TB was not negatively impacting their family relationships increased from $60 \%$ at baseline to $71 \%$ at follow-up. Following family-based counseling, there were notable improvements in interpersonal relationships within families, increased family support for TB patients and improved adherence to treatment which resulted in zero "default" rate for treatment outcomes among the family-based counseling participants compared to $5 \%$ "default" in the comparison group ( $\mathrm{p}=$ 0.07). Conclusion: The evaluation showed that a low-cost one-time familybased educational intervention with a psychological component can be effective in improving treatment outcomes of TB patients.
\end{abstract}

\section{Keywords}

Tuberculosis, Family Support, Counseling 


\section{Introduction}

Drug-susceptible TB can be treated with four standard first-line anti-TB drugs. Misuse or mismanagement of these drugs may lead to multidrug resistant (MDR) TB [1]. Up to half of people with tuberculosis (TB) do not complete their treatment worldwide [2]. The National Tuberculosis Control Center (NTCC) organizes TB treatment in Armenia following the WHO recommendations for patients to take TB medications under observation of TB physicians or nurses, with an optimal dosing frequency of six days per week for new TB patients during both intensive and continuous phases of treatment [3] [4]. Directly Observed Therapy (DOT) aimed to facilitate patients' adherence to their treatment by scheduling the on-time medication intake, leading to reduction of the drug-resistance development [4] [5]. The responsibility for direct supervision of patients taking TB medication primarily falls on inpatient and outpatient health facilities [3].

Treatment adherence has been associated with various factors, including disease-related knowledge, health care satisfaction, and social support [4] [6]. Interventions that had educational components, demonstrated enhanced treatment adherence among patients [7] [8]. As a result, those patients complete their treatment with better outcomes and improved quality of life [9]. Physicians contribute to patients' poor adherence by failing to adequately explain the benefits and side effects of a medication [10]. A number of studies show that involving social workers or family members (FM) in some capacity in TB treatment is an effective way for improving patients' adherence [11] [12] [13] [14]. Empowering patients and focusing on TB education positively affects the process and outcome of TB care. A people-centered approach extends the focus of treatment from disease to person [15].

This pilot intervention was based on a low-cost family-centered model, which included a 90-minute intervention that could empower and educate FMs and facilitate their inclusion in the management of TB patients' treatment, to assure that TB drugs are taken regularly and that side-effects and symptoms are monitored and reported to TB physicians during the continuation outpatient phase of treatment. The objective of this pilot study was to explore the feasibility and the effectiveness of the applied intervention and to test evaluation instruments providing valuable insights for further larger scale research. If proven successful, family-based interventions could be integrated into the current TB system, ultimately contributing to the reduction of failed $\mathrm{TB}$ treatment and rates of drug-resistance, of the spread of $\mathrm{TB}$, and diminishing the stigma of $\mathrm{TB}$ in the community.

\section{Methods}

Targeting the study objectives we utilized both qualitative and quantitative research methods. The pre-intervention qualitative research provided directions for designing a brief effective family-based counseling (FBC) intervention in- 
cluding the development of appropriate FBC materials and followed by FBC intervention and its quantitative evaluation.

\subsection{Pre-Intervention Qualitative Study}

We conducted focus group discussions and semi-structured in-depth interviews with key informants who were identified using purposive and convenience sampling methods. The choice of informants was based on their experience and expertise in TB services. Five groups of participants took part in the qualitative study: 1) physicians, 2) nurses, 3) FMs of TB patients, 4) TB patients, and 5) experts from NTCC, Médecins Sans Frontières France, and the Armenian Red Cross Society (ARCS).

A semi-structured in-depth interview and focus group discussion guides were developed in English and translated into Armenian and pretested. TB physicians and nurses from the outpatient centers obtained TB patients' permission for the research team to contact them and FMs. Each focus group (FG) and in-depth interviews had a trained moderator and a note-taker. These roles were rotated among the research team members. Audio-recording was possible only with permission of participants; if a participant did not want to be audio-recorded, only written notes were taken. For all participants, anonymity and confidentiality were fully respected.

Nineteen people participated in four focus groups discussions that were supplemented with five in-depth interviews. TB patient or FM of TB patient participating in the pre-intervention research were not included in the evaluation of the intervention. To optimize the program design for both urban and rural regions, and to account for differences in TB services and family culture in different regions, we invited representatives from both Yerevan (capital of Armenia) and provinces to participate in the qualitative research. Fifteen participants were from Yerevan and four from provinces, with the mean age of 41 . The average number of years of professional experience of TB specialists and TB experts was 12 years. About one-fifth of the study participants were males. The mean duration of the focus group discussions was 68 minutes and the mean duration of in-depth interviews was 50 minutes.

For the analysis we used mixed-conventional inductive and directed deductive content analysis techniques [16] [17].

\subsection{Development of Family-Based Counseling Materials}

The training package included adapted materials from the US Center for Disease and Prevention, WHO, ARCS, and the NTCC of the Ministry of Health of Armenia [18] [19]. The training package included the FBC educational materials, a flipchart for counseling support, and brochures for distribution to TB patients and their FMs during the FBC intervention. In addition, we developed a special guide to assess the emotional state of $\mathrm{TB}$ patients, their needs and relations with FMs prior to the FBC session. 


\subsection{Family-Based Counseling Intervention}

This people-centered pilot intervention involved adult TB patients and their FMs from Shirak, Kotayk and Aragatsotn provinces. We selected these three provinces out of 11 (including the capital city Yerevan) in Armenia for the pilot FBC based on their relatively high TB and MDR-TB notification rates, the number of TB patients, and absence of concurrent TB training programs for patients and their families. All Armenian-speaking drug-susceptible TB patients who were 18 years-of-age or older at the first three months of the continuation outpatient phase of treatment and their FMs (selected by patients) were eligible to participate in the 90-minutes FBC. Eight patients and 18 FMs from Aragatsotn, 24 patients and 37 FM from Kotayk, and 23 patients and 26 FM from Shirak provinces participated in the intervention (55 patients and $81 \mathrm{FMs}$ ).

The counseling sessions were conducted in a setting and at a time most convenient for the TB patients. Majority of the sessions happened in the homes of TB patients, at times when visitors were least expected. Only few sessions were conducted in the TB outpatient centers, at the request of the TB patients and upon permission of the centers' administration and the TB physicians.

\subsection{Intervention Evaluation}

We conducted a baseline-follow-up panel survey of TB patients and their FMs, to evaluate the influence of the FBC on participants' knowledge, stigma, and socio-psychological support within the family of the TB patients. All those TB patients and their FMs who could read and write in Armenian, and were 18 years old and above were eligible to participate in the evaluation surveys. Nine participants of the intervention who were younger than 18 or could not read and write in Armenian were excluded from the evaluation. Only three eligible people refused to participate. Out of 54 eligible TB patients 52 participated in the evaluation; out of 72 eligible FMs of TB patients 57 participated in both baseline and follow-up surveys (study power $=0.90$ ) [20].

We developed two study instruments based on the FBC package contents and similar instruments used for other TB assessments to collect information from TB patients and from FMs [21]. The FBC trainers (teams of psychologists and TB-nurses) conducted the baseline (prior to FBC session) and follow-up (one month after the session) surveys.

We used SPSS 16 statistical software for all analyses except for applications of exact tests where StatXact 10 was utilized. To test differences in proportions between baseline and follow-up, we used the McNemar's test for two related samples. To test the difference between means of continuous variables at baseline and follow-up we used the paired t-test. To assess the level of knowledge of TB patients and FMs about TB before and after the counseling session, we calculated the cumulative knowledge score based on 28 knowledge items and the mean percent cumulative knowledge score based on the proportion of correct answers. To test differences in treatment outcomes between FBC study participants and 
TB patients of the group the unconditional-margins Barnard's one-sided exact test was applied [22].

To compare outcome measures between the TB-patient FBC study participants and a group of TB patients who were not exposed to pilot intervention we abstracted data from the NTCC database on TB patients' "success", "failed", "death", and "lost-to-follow-up" treatment outcomes rates. We compared the official TB treatment outcomes data for the FBC study participants (4th quarter 2012) with the official data on treatment outcomes for two comparable groups of drug-susceptible TB patients of 18 years-of-age or older from the same provinces (Aragatsotn, Kotayk, and Shirak) who received their continuation outpatient phase of treatment in the 4th quarter of 2011 and 2013.

The American University of Armenia Institutional Review Board approved the study for compliance with locally and internationally accepted ethical standards. Informed consent was sought from each study participant. Their privacy was adequately protected and the data was maintained confidentially.

\section{Results}

\subsection{Pre-Intervention Qualitative Study}

The qualitative research showed that most patients and their families were interested in learning more about TB than what was usually provided to them. The study participants emphasized the importance of rigorously maintaining confidentiality of the patients and families, due to stigma of TB in the communities, and understanding the structure and functioning of the family and awareness of the emotional states of TB patients and their FMs for effective counseling. They believed that the choice of the candidates in the family for supporting the TB patients should depend on the personal relationships and power structures within that family.

\subsection{Intervention Evaluation}

After selection, overall, $55 \mathrm{~TB}$ patients and 81 patient-selected FMs consented to participate in the FBC intervention (Figure 1). The majority of those TB patients who refused to take part in a FBC session were younger males leading to a statistically significant different mean age for the participants vs. refusals $(p=0.02)$ (Table 1). For all other TB characteristics, including type of TB (pulmonary/extra-pulmonary), number of treatments (new/retreated cases), and infectivity status at the beginning of the treatment (smear positive/smear negative) the participants were similar to those who refused to participate (Table 1). The overall response rate for completion of both surveys was $94.6 \%$ among TB patients and $70.4 \%$ among FMs (Figure 1).

The majority of the participants in both study groups had high school education (63.5\% of TB patients and $68.4 \%$ of FMs), and about $36.5 \%$ of patients and $31.6 \%$ of family members had professional/technical and university education. 




Figure 1. Flow diagram of the study population in the Family Based Counseling (FBC) intervention/evaluation. (a. One FBC participant could not complete the surveys because lacking literacy in Armenian).

Table 1. Comparisons of TB patients and treatment outcomes based on national databases for the 4th quarter 2011 and 2013 and participants of Family Based Counseling (FBC) (4th quarter 2012).

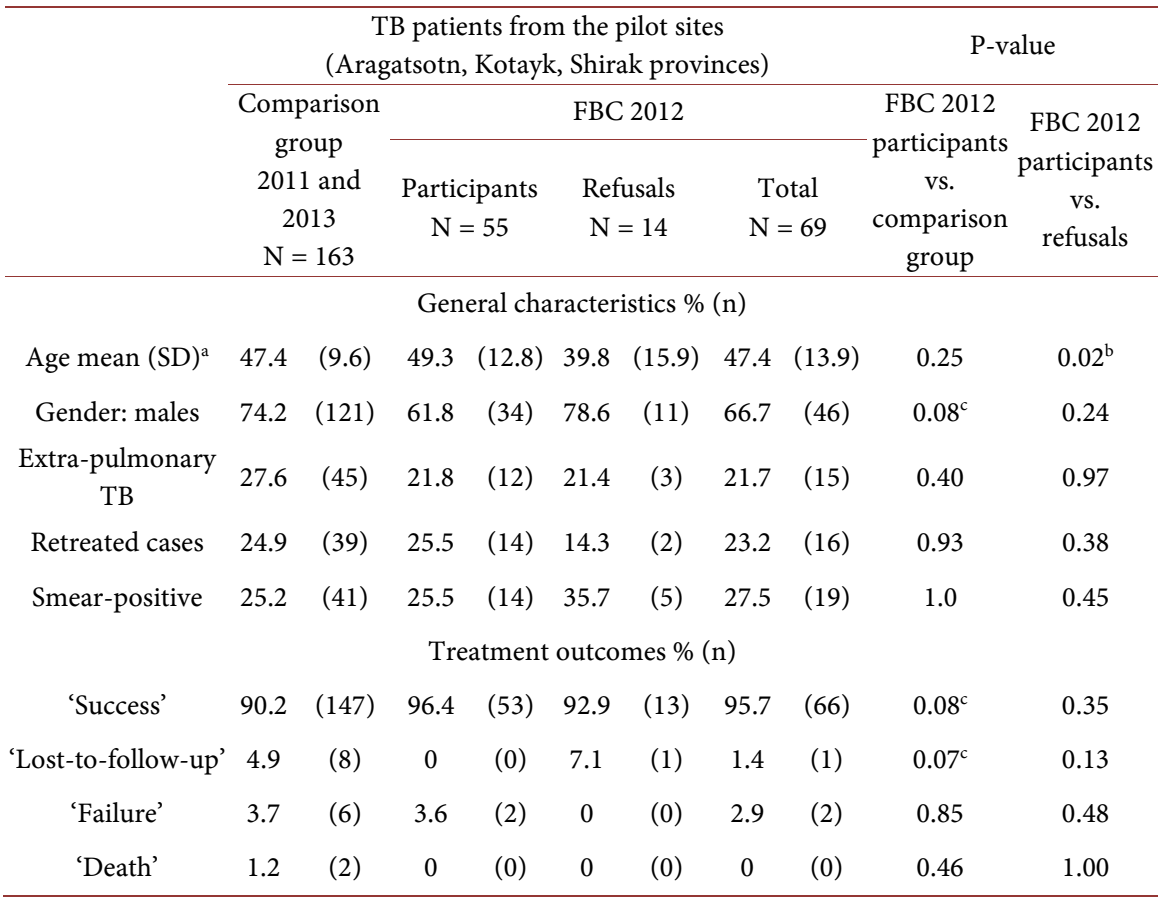

a. SD-Standard Deviation b. Statistically significant difference $\mathrm{p} \leq 0.05 \mathrm{c}$. Marginal statistically significant difference $0.05<\mathrm{p} \leq 0.1$ 


\section{- Knowledge}

The proportions of correct answers increased for almost all questions in both study groups at the follow-up one month after FBC. A statistically significant improvement in the proportion of correct answers was recorded for nine questions among TB patients and 11 questions among FMs out of 28 questions (McNemar's $\mathrm{p}<0.05$ ). The mean cumulative knowledge score for TB patients significantly increased from 19.3 at baseline to 21.9 at follow-up one month later ( $\mathrm{p}<0.001$ ), representing an increase from $68.9 \%$ to $78.2 \%$ percent score. For FMs the score increased from 18.0 at baseline to 21.7 at follow-up $(\mathrm{p}<0.001)$, increasing from $64.3 \%$ to $77.5 \%$ (Table 2). TB patients and FMs had scores similar to each other at both baseline and follow-up. Socio-demographic characteristics of the study participants such as age, education, region of residence, and monthly family expenditures were not associated with study participants' TB-related cumulative knowledge scores and changes in cumulative knowledge scores.

\section{- Stigma}

The proportion of those who believed that TB was not negatively affecting personal relationships between the non-infectious TB patients and their families increased, though non-statistically significantly, from $59.5 \%$ at baseline to $67.3 \%$ at follow-up for TB patients and from $60.9 \%$ to $73.2 \%$ for FMs. There was also a substantial but non-statistically significant increase in the proportion of TB FMs who thought that TB patients did not want to hide their TB diagnosis from their family from $74.5 \%$ at baseline to $83.9 \%$ at follow up. This percentage did not change substantially among the TB patients.

\section{- Family support}

The patients and their FMs were asked to evaluate the level of family support on a scale from 1 to 5 . TB patients reported a mean score for family support of 4.90 at baseline and an increase to 4.98 at follow-up, while FMs reported a mean of 4.78 at baseline and an increase 4.81 at follow-up. The reported differences in family support scores between baseline and follow-up were not statistically significant.

\section{- Treatment outcomes}

Two TB patient comparison groups from the 4th quarter of 2011 and the 4th quarter of 2013 were identified based on the same eligibility criteria as the TB patient group who participated in FBC. Demographic characteristics and treatment outcomes of these two groups were similar, that is why we collapsed these two groups of patients into one comparison group.

Demographic characteristics of the FBC TB patient study population and the TB patient comparison group were also similar. Though the FBC TB patient group had lower "failure" and "death" outcome rates than the comparison group, none of the results achieved statistical significance. Improvements in "success" and "lost-to-follow-up" treatment outcomes for the FBC TB group showed marginal statistical significance over the comparison group $(\mathrm{p}=0.08$ and $\mathrm{p}=0.07$ respectively) (Table 1$)$. 
Table 2. TB knowledge by TB patients and family members (FMs) with baseline/follow-up comparison.

\begin{tabular}{|c|c|c|c|c|c|c|c|c|c|c|}
\hline \multirow{3}{*}{ Items } & \multicolumn{5}{|c|}{ TB patients } & \multicolumn{5}{|c|}{$\mathrm{FMs}^{\mathrm{a}}$} \\
\hline & \multicolumn{2}{|c|}{ Baseline $\mathrm{N}=52$} & \multicolumn{2}{|c|}{ Follow-up N = 52} & \multirow{2}{*}{$\begin{array}{c}\mathrm{P} \\
\text { value }\end{array}$} & \multicolumn{2}{|c|}{$\begin{array}{l}\text { Baseline } \\
\mathrm{N}=57\end{array}$} & \multicolumn{2}{|c|}{$\begin{array}{l}\text { Follow-up } \\
\mathrm{N}=57\end{array}$} & \multirow{2}{*}{$\begin{array}{c}\mathrm{P} \\
\text { value }\end{array}$} \\
\hline & $\%$ & $\mathrm{n}$ & $\%$ & $\mathrm{n}$ & & $\%$ & $\mathrm{n}$ & $\%$ & $\mathrm{n}$ & \\
\hline \multicolumn{11}{|c|}{ A person can get TB through: } \\
\hline Handshakes & 33.3 & (17) & 2.0 & (1) & $<0.01^{\mathrm{b}}$ & 24.1 & (13) & 3.7 & (2) & $0.01^{\mathrm{b}}$ \\
\hline The air when a TB patient coughs/sneezes & 86.3 & $(44)$ & 96.1 & $(49)$ & 0.13 & 63.0 & $(34)$ & 96.3 & $(52)$ & $<0.01^{\mathrm{b}}$ \\
\hline Direct contact with $\mathrm{TB}$ patients & 51.0 & $(26)$ & 41.2 & $(21)$ & 0.36 & 42.1 & $(24)$ & 61.1 & $(33)$ & 0.69 \\
\hline Touching items in public places & 43.1 & $(22)$ & 19.6 & $(10)$ & $0.01^{\mathrm{b}}$ & 24.1 & (13) & 4.8 & (8) & 0.33 \\
\hline Using same sanitary utensils & 45.1 & (23) & 7.8 & (4) & $<0.01^{\mathrm{b}}$ & 38.9 & $(21)$ & 11.1 & (6) & $<0.01^{\mathrm{b}}$ \\
\hline Sharing same platter & 52.9 & $(27)$ & 7.8 & (4) & $<0.01^{\mathrm{b}}$ & 50.0 & $(27)$ & 7.4 & (4) & $<0.01^{\mathrm{b}}$ \\
\hline \multicolumn{11}{|c|}{ The signs of TB: } \\
\hline Fever & 65.4 & $(34)$ & 73.1 & $(38)$ & 0.39 & 69.6 & $(39)$ & 78.6 & $(44)$ & 0.33 \\
\hline Cough, lasting longer than 3-weeks & 57.7 & $(30)$ & 73.1 & $(38)$ & 0.13 & 50.0 & $(28)$ & 75.0 & $(42)$ & $<0.01^{\mathrm{b}}$ \\
\hline Coughing up blood & 76.9 & $(40)$ & 75.0 & $(39)$ & 1.00 & 58.9 & $(33)$ & 62.5 & $(35)$ & 0.80 \\
\hline Weight loss & 84.6 & $(44)$ & 86.5 & $(45)$ & 1.00 & 62.5 & $(35)$ & 83.9 & $(47)$ & $<0.01^{\mathrm{b}}$ \\
\hline Chest pain & 61.5 & $(32)$ & 69.2 & $(36)$ & 0.45 & 51.8 & $(29)$ & 69.6 & $(39)$ & $0.03^{\mathrm{b}}$ \\
\hline Difficulty breathing/not enough air & 59.6 & $(31)$ & 65.4 & $(34)$ & 0.63 & 53.6 & $(30)$ & 62.5 & $(35)$ & 0.38 \\
\hline Weakness & 80.8 & $(42)$ & 76.9 & $(40)$ & 0.79 & 64.3 & $(36)$ & 76.8 & $(43)$ & 0.21 \\
\hline Sweating & 82.7 & $(43)$ & 73.1 & $(38)$ & 0.27 & 69.6 & $(39)$ & 71.4 & $(40)$ & 1.00 \\
\hline \multicolumn{11}{|c|}{ A person in the infective stage of $\mathrm{TB}$ can prevent the spread of disease through: } \\
\hline Immediate treatment & 75.0 & $(39)$ & 82.7 & $(43)$ & 0.39 & 83.6 & $(46)$ & 87.3 & $(48)$ & 0.77 \\
\hline Ventilating room frequently & 75.0 & $(39)$ & 84.6 & $(44)$ & 0.27 & 69.1 & $(38)$ & 76.4 & $(42)$ & 0.65 \\
\hline Covering mouth \& nose when cough/sneeze & 82.7 & $(43)$ & 84.6 & $(44)$ & 1.00 & 76.4 & $(42)$ & 92.7 & $(51)$ & $0.01^{\mathrm{b}}$ \\
\hline Wearing mask & 73.1 & $(38)$ & 53.8 & $(28)$ & $0.02^{\mathrm{b}}$ & 54.5 & $(30)$ & 60.0 & $(33)$ & 0.70 \\
\hline Avoiding use of public transportation & 44.2 & $(23)$ & 30.8 & $(16)$ & 0.17 & 29.1 & $(16)$ & 26.2 & $(28)$ & 0.45 \\
\hline Avoiding shaking hands & 34.6 & $(18)$ & 1.9 & (1) & $<0.01^{\mathrm{b}}$ & 27.3 & $(15)$ & 9.1 & (5) & $0.03^{\mathrm{b}}$ \\
\hline Washing hands frequently & 26.9 & $(14)$ & 46.2 & $(24)$ & $<0.01^{\mathrm{b}}$ & 63.6 & $(35)$ & 45.5 & $(25)$ & $0.02^{\mathrm{b}}$ \\
\hline Interacting with people in confined areas & 28.8 & $(15)$ & 5.8 & (3) & $<0.01^{\mathrm{b}}$ & 23.6 & $(13)$ & 3.6 & (2) & $<0.01^{\mathrm{b}}$ \\
\hline \multicolumn{11}{|c|}{ The best method of TB treatment: } \\
\hline $\begin{array}{l}\text { Taking prescribed drugs every day in the } \\
\text { presence of doctor or nurse }\end{array}$ & 93.5 & $(43)$ & 97.8 & $(45)$ & 0.50 & 93.8 & $(45)$ & 97.9 & $(47)$ & 0.50 \\
\hline \multicolumn{11}{|c|}{ The consequences of not following the treatment scheme correctly: } \\
\hline Remaining seek for a longer period & 80.9 & $(38)$ & 89.4 & $(42)$ & 0.39 & 78.2 & $(43)$ & 83.6 & $(46)$ & 0.63 \\
\hline Spreading tuberculosis to other people & 74.5 & $(35)$ & 76.6 & $(36)$ & 1.00 & 70.9 & $(39)$ & 70.9 & $(39)$ & 1.00 \\
\hline Developing drug-resistant tuberculosis & 55.3 & (26) & 76.6 & $(36)$ & $0.04^{\mathrm{b}}$ & 52.7 & (29) & 63.6 & (35) & 0.21 \\
\hline Developing more severe $\mathrm{TB}$ case & 76.6 & $(36)$ & 68.1 & $(32)$ & 0.45 & 72.7 & $(40)$ & 74.5 & $(41)$ & 1.00 \\
\hline No changes & 83.0 & $(39)$ & 97.9 & $(47)$ & $0.04^{\mathrm{b}}$ & 92.7 & $(51)$ & 98.2 & $(54)$ & 0.38 \\
\hline Mean cumulative knowledge score & \multicolumn{2}{|c|}{19.3} & \multicolumn{2}{|c|}{21.9} & $<0.01^{\mathrm{b}}$ & \multicolumn{2}{|c|}{18.0} & \multicolumn{2}{|c|}{21.7} & $<0.01^{\mathrm{b}}$ \\
\hline
\end{tabular}

a FMs-Family Members. b Statistically significant difference $\mathrm{p} \leq 0.05$. 


\section{Discussion}

This brief pilot intervention aimed to improve knowledge on TB of TB patients and their FMs, provide psychological support and empower the patients and their FMs during the treatment period. The evaluation of the intervention showed that TB patients' and FMs' knowledge on TB and the importance of adherence to the treatment plan substantially improved after the counseling session. The reductions in TB-associated stigma reported by TB patients and their FMs were substantial but not statistically significant. Lack of statistical significance could be due to a small sample size in this pilot study; questions were mainly directed towards stigma in the community and not within the family. Thus, the questions were probably less sensitive to measuring changes in stigma within the family.

Families reported highly supportive practices and behaviors toward FMs with TB at baseline and showed substantial though not statistically significant improvements at follow-up. Though close family ties and strong family support are highly valued in Armenia [23], particularly in rural regions, it is possible that study participants inflated their scores for some indicators of support at baseline to impress the FBC trainers, leading to a smaller magnitude of change at follow-up. While the counseling session was designed to equip the families with knowledge and skill-sets to improve family support, the survey might not have been sufficiently sensitive to capture the more subtle changes in the quality of family support.

The comparison of treatment outcomes for TB patients who participated in the FBC session and those who did not, suggested long-term success of the intervention. Providing TB patients and families with knowledge about tuberculosis disposing of common TB myths, increasing support within families, empowering TB patients and their FMs, and decreasing TB-related stigma within families might lead to better treatment outcomes among TB patients. Among unsuccessful TB treatment outcomes ("failed", "death", and "lost-to-follow-up"), "failed" and "death" are more directly associated with co-morbidities and clinical factors [24] [25], while "lost-to-follow-up" is more directly associated with non-adherence and patient behaviors [15]. However, "lost-to-follow-up" elevates the risk of severity of disease, leading to higher rates of TB "death" and "failure" and increasing the likelihood of drug resistant TB. There were no "lost-to-follow-up" in the FBC intervention group (0/55), compared to 5\% (8/163) in the comparison group and 7\% (1/14) among FBC refusals, suggesting improved outcomes for the FBC intervention.

Few studies had comprehensively assessed the role and the impact of counseling on TB patients' treatment outcomes, especially during continuation phase of treatment. A study conducted in Pakistan showed that lost-to-follow-up of TB patients is less when counseled [26]. Their findings were in line with our results showing that patients who received TB knowledge and support, started to believe that their tuberculosis was curable while their FMs felt that it helped them 
to change their negative attitudes towards TB patients [27]. A recent Ethiopian study concluded that counseling might facilitate treatment adherence of HIV co-infected TB patients [28], and a Cochrane review [7] confirmed that counseling and education promote adherence to TB treatment and latent TB infection treatment when the context of information provided and the assessment of the causality for poor adherence are thoroughly considered.

Our study findings also support the concept of effective combination of supervised treatment and the counseling for TB patients. As other authors believe that providing additional education and counseling to TB patients does not diminish the importance of directly observed treatment [27] [29] we also confirm that counseling can be used as a reinforcing tool for improving TB patients' treatment adherence by aligning and motivating their behavior toward compliance with TB treatment standards particularly in resource-poor countries [29].

\section{Limitations}

In this pilot intervention some of the questions to measure TB-related stigma in the study tool may not have been sufficiently sensitive to the intervention, given that the questions were more directed towards stigma in the larger community and not within TB-afflicted families. However, regardless of the emphasis of the questions, there were still substantial improvements on measures of stigma, though not statistically significant given the limited sample size.

\section{Conclusion}

Changing beliefs among TB patients and their families to improve TB treatment outcomes can be enhanced through a single 90-minute intervention involving TB patient and family counseling and education by a trained nurse and a psychologist. Empowering TB patients and their family members can elevate their understanding and enhance adherence to treatment, leading to reductions in "lost-to-follow-up" rates which can lead to reduced rates of drug-resistant TB.

\section{Acknowledgements}

This work was financially supported by the Armenian Medical Fund, USA.

We want to express our gratitude to the trainers who conducted the groundwork that changed the lives of ТB patients and their families. Authors thank Ruzanna Grigoryan, Marianna Koshkakaryan, Meri Tadevosyan, and Hripsime Martirosyan for their support during the study implementation, the National TB Control Center of the Ministry of Health of the Republic of Armenia and the polyclinic administrators, who provided their constant and committed support throughout the study.

\section{References}

[1] Donald, P. and Helden, P.D. (2009) The Global Burden of Tuberculosis-Combating Drug Resistance in Difficult times. The New England Journal of 
Medicine, 360, 2393-2395. https://doi.org/10.1056/NEJMp0903806

[2] Volmink, J. and Garner, P. (2000) Interventions for Promoting Adherence to Tuberculosis Management. Cochrane Database of Systematic Reviews, 4, Article ID: CD000010.

[3] Aït-Khaled, N., Alarcón, E., Armengol, R., et al. (2010) Management of Tuberculosis. A Guide to the Essentials of Good Practice. 6th Edition, International Union against Tuberculosis and Lung Disease, Paris.

[4] Aziz, M.A. and Wright, A. (2005) The World Health Organization/International Union against Tuberculosis and Lung Disease Global Project on Surveillance for Anti-Tuberculosis Drug Resistance: A Model for Other Infectious Diseases. Clinical Infectious Diseases, 41, S258-S262. https://doi.org/10.1086/430786

[5] Vink, K., Colombani, P., Mosneaga, A., Dara, M., Dauby, C., Hennig, C., Bates J. and Khachatryan, S. (2005) Tuberculosis Assessment Mission to Armenia. WHO Regional Office for Europe, Copenhagen.

[6] Barnhoorn, F. and Adriaanse, H. (1992) In Search of Factors Responsible for Noncompliance among Tuberculosis Patients in Wardha District, India. Social Science \& Medicine, 34, 291-306. https://doi.org/10.1016/0277-9536(92)90271-Q

[7] M'Imunya, J.M., Kredo, T. and Volmink, J. (2012) Patient Education and Counselling for Promoting Adherence to Treatment for Tuberculosis. Cochrane Database of Systematic Reviews, 5, Article ID: CD006591.

[8] Morisky, D.E., Malotte, K., Davidson, P., et al. (1990) A Patient Education Program to Improve Adherence Rates with Antituberculosis Drug Regimens. Health Education Quarterly, 17, 253-267. https://doi.org/10.1177/109019819001700303

[9] Guo, N., Marra, F. and Marra, C.A. (2009) Measuring Health-Related Quality of Life in Tuberculosis: A Systematic Review. Health and Quality of Life Outcomes, 7, 14. https://doi.org/10.1186/1477-7525-7-14

[10] Mitchell, A.J. and Selmes, T. (2007) Why Don't Patients Take Their Medicine? Reasons and Solutions in Psychiatry. Advances in Psychiatric Treatment, 13, 336-346. https://doi.org/10.1192/apt.bp.106.003194

[11] Newell, J.N., Baral, S.C., Pande, S.B., Bam, D.S. and Malla, P. (2006) Family-Member DOTS and Community DOTS for Tuberculosis Control in Nepal: Cluster-Randomised Controlled Trial. The Lancet, 367, 903-909. https://doi.org/10.1016/S0140-6736(06)68380-3

[12] Akkslip, S., Rasmithat, S., Maher, D. and Sawert, H. (1999) Direct Observation of Tuberculosis Treatment by Supervised Family Members in Yasothorn Province, Thailand. The International Journal of Tuberculosis and Lung Disease, 3, 1061-1065.

[13] Garner, P. and Volmink, J. (2006) Families Help Cure Tuberculosis. The Lancet, 367, 878-879. https://doi.org/10.1016/S0140-6736(06)68356-6

[14] Maciel, E.L.N., Guidoni, L.M., Brioshi, A.P., et al. (2010) Household Members and Health Care Workers as Supervisors of Tuberculosis Treatment. Revista de Saúde Pública, 44, 339-343. https://doi.org/10.1590/S0034-89102010000200015

[15] Mezzich, J.E., Appleyard, J., Botbol, M., et al. (2014) Summary Report of the Seventh Geneva Conference on Personcentered Medicine. The International Journal of Person Centered Medicine, 4, 139-144.

[16] Curry, L.A., Nembhard, I.M. and Bradley, E.H. (2009) Qualitative and Mixed Methods Provide Unique Contributions to Outcomes Research. Circulation, 119, 1442-1452. https://doi.org/10.1161/CIRCULATIONAHA.107.742775 
[17] Meyer, J. (2000) Qualitative Research in Health Care: Using Qualitative Methods in Health Related Action Research. British Medical Journal, 320, 178-181. https://doi.org/10.1136/bmj.320.7228.178

[18] CDC (2008) Self-Study Modules on Tuberculosis. U.S. Department of Health and Human Services. Centers for Disease Control and Prevention, National Center for HIV/AIDS, Viral Hepatitis, STD, and TB Prevention, Division of Tuberculosis Elimination, Atlanta.

[19] ARC (2011) Tuberculosis (TB) Teaching Flip Chart. Armenian Red Cross Society, Yerevan.

[20] Hertzog, M.A. (2008) Considerations in Determining Sample Size for Pilot Studies. Research in Nursing \& Health, 31, 180-191. https://doi.org/10.1002/nur.20247

[21] Truzyan, N., Crape, B., Grigoryan, R., Martirosyan, H. and Petrosyan, V. (2015) Increased Risk for Multidrug-Resistant Tuberculosis in Migratory Workers, Armenia. Emerging Infectious Diseases, 21, 474-476. https://doi.org/10.3201/eid2103.140474

[22] Andres, A.M. and Mato, A.S. (1994) Choosing the Optimal Unconditioned Test for Comparing Two Independent Proportions. Computational Statistics \& Data Analysis, 17, 555-574. https://doi.org/10.1016/0167-9473(94)90148-1

[23] Miller, D. and Miller, L.T. (1993) Armenian Families, in Survivors: An Oral History of the Armenian Genocide. University of California Press, Berkeley.

[24] Endris, M., Moges, F., Yeshambel Belyhun, Y., et al. (2014) Treatment Outcome of Tuberculosis Patients at Enfraz Health Center, Northwest Ethiopia: A Five-Year Retrospective Study. Tuberculosis Research and Treatment, 2014, Article ID: 726193. https://doi.org/10.1155/2014/726193

[25] Albuquerque, M.F., Ximenes, R.A., Lucena-Silva, N., et al. (2007) Factors Associated with Treatment Failure, Dropout, and Death in a Cohort of Tuberculosis Patients in Recife, Pernambuco State, Brazil. Cadernos de Saúde Pública, 23, 1573-1582. https://doi.org/10.1590/S0102-311X2007000700008

[26] Raza, S., Sarfaraz, M. and Ahmad, M. (2012) Practice of Family and Non-Family Based Directly Observed Treatment for Tuberculosis in Pakistan: A Retrospective Cohort Study. The Health, 3, 39-44.

[27] Liefooghe, R., Suetens, C., Meulemans, H., Moran, M.B. and De Muyncket, A. (1999) A Randomised Trial of the Impact of Counselling on Treatment Adherence of Tuberculosis Patients in Sialkot, Pakistan. International Journal of Tuberculosis and Lung Disease, 3, 1073-1080.

[28] Gebremariam, K.M., Bjune, G.A. and Frich, J.C. (2010) Barriers and Facilitators of Adherence to TB Treatment in Patients on Concomitant TB and HIV Treatment: A Qualitative Study. BMC Public Health, 10, 651.

[29] Thiam, S., LeFevre, A.M., Hane, F., et al. (2007) Effectiveness of a Strategy to Improve Adherence to Tuberculosis Treatment in a Resource-Poor Setting. A Cluster Randomized Controlled Trial. JAMA, 297, 380-386. 